\title{
A Review on Timing \& Synchronization Technique in OFDM
}

\author{
Shweta Kolwal ${ }^{1}$, Sandeep Agrawal ${ }^{2}$ \\ ${ }^{1}$ PG Student, ECE Department, MIT, Ujjain \\ ${ }^{2}$ Ass. Professor, Electronics \& Communication Engg. , MIT Ujjain
}

\begin{abstract}
The aim of this paper is to survey and compare previous methods for Data-aided timing and frequency synchronization of OFDM System.Synchronization has been one of the crucial research topics in orthogonal frequency division multiplexing (OFDM) system because of its sensitivity to the timing and frequency errors [1]. To guarantee the fast and accurate data transmission, the Inter Symbol Interference (ISI) and Inter Carrier Interference (ICI) caused in the transmission have to be eliminated as much as possible. ORTHOGONAL frequency-division multiplexing (OFDM) has been of major interest for both wireline-based and wireless applications [1]-[3] due to its highdata rate transmission capability and its robustness to multipathdelay spread. However, OFDM systems are much more sensitive to synchronization errors than single carrier systems [4],[5]. This paper summarizes various methods to cater this synchronization problem.
\end{abstract}

Keywords: OFDM, ICI, ISI, MIMO, Chu sysmbol

\section{Introduction to PAPR}

The combination of multiple-input multiple-output (MIMO) signal processing with orthogonal frequency division multiplexing (OFDM) [1]-[2] is regarded as a promising solution for the next generation wireless communication systems operating in frequency selective fading environments [3]. However, MIMO OFDM systems are very sensitive to synchronization errors. Several approaches have been proposed for time and frequency synchronization [4][9].Several approaches have been proposed for estimating thetime and frequency offset either jointly [6]-[8] or individually.See [9]-[12] for further notes on frequencyoffset estimationand to [13]-[16] on timing estimation. There are of numerousother relevant contributions in the literature and a good discus-sion on them can be found in the recent paper [28].

Most frequency and timing estimation methods exploit theperiodic nature of the time-domain signal by using a cyclicprefix [6], [8], [10], [13], or by designing the training symbolhaving repeated parts [7], [9], [12]. Regarding frequencyestimation, in [7], a training symbol containing two identicalhalves is used and the frequency acquisition range is 1subcarrier spacing. In order to increase the frequency capturerange, a second training symbol is employed. A frequencyestimation scheme improving the solution of [7] is proposedin [12], where only one training symbol having identicalparts is required and the frequency acquisition range issubcarrier spacing. A robust timing synchronization schemeusing a training symbol having two identical parts has also beenproposed in [7]. However, the timing metric plateau inherentin [7] results in a large timing offset estimation variance. Thistiming metric plateau can be eliminated and, hence, the timingoffset estimation variance can be reduced by designing thetraining symbol such that it gives a more pronounced timingmetric trajectory [15]. For single carrier systems, in [17], theframe synchronization performance has been considerablyimproved by designing the training preambles to give a sharperpeak timing metric trajectory.

All of the above OFDM synchronization methods are associated with one or more of the following limitations or drawbacks: have a limited range of operation, address only one task,have a large estimation variance, lack robust sync detection ca-pability, and require extra overheads.

\section{Timing and Frequency Synchronizationfor of DM, Using Only One Training Symbol}

In this case, the training symbol isdesigned to have a sharp timing metric trajectory. In choosingthe timing metric, a robust sync detection capability has to betaken into consideration. We design the training symbol to becomposed of repeated identical parts with possible sign inver-sions. Our choice of this type of training symbol is based onthe following reasons. The periodicity or repetitive nature of thetraining symbol equips timing synchronization with robustnessagainst frequency offsets. Having multiple identical parts givesthe benefit of using the same training symbol for frequency synchronization, which can handle large frequency offsets. By de-signing the signs of the identical parts to give the sharpest pos-sible timing metric trajectory, the timing offset estimation canbe improved. Both OFDM-type training symbols (frequencydomain (FD) training) and single-carrier-type training blocks(time domain (TD) training) are investigated.

In this synchronization scheme, which provides bothtiming and frequency estimation as well as channel estimates.A specifically designed training symbol is used for bothtiming and frequency synchronization. Channel estimationbased on the designed training symbol is also incorporated inorder to give fine-timing and frequency-offset estimates. Fine synchronization can also be iteratively improved. The impact ofusing only one training symbol for both timing and frequencysynchronization is discussed and a number of approaches areproposed for further performance 


\section{International Journal of Science and Research (IJSR) \\ ISSN (Online): 2319-7064}

Index Copernicus Value (2015): 78.96 | Impact Factor (2015): 6.391

improvement. The syncdetection performance, timing synchronization performance,frequency synchronization performance, and bit-error-rate(BER) performance of the proposed method is evaluated bycomputer simulations.

In OFDM systems, the main synchronization parameters tobe estimated are a sync flag indicating the presence of the signal(especially for burst-mode transmission), the starting time ofthe FFT window (timing synchronization), the frequency offsetdue to the inaccuracies of the transmitter and receiver oscilla-tors, and the Doppler shift of the mobile channel, as well as thechannel estimates if coherent reception is adopted. The sync flagcan be generated by automatic gain control (e.g., ramp-up indi-cation via power measurement and threshold decision) or usinga training symbol (which can also be used for timing synchro-nization and possibly frequency synchronization). For the lattercase, the same metric used for timing synchronization may beused together with the threshold decision, in order to generatethe sync flag. After detecting the presence of the signal, the othersync parameters are estimated.

The flowchart below explains the complete process :-

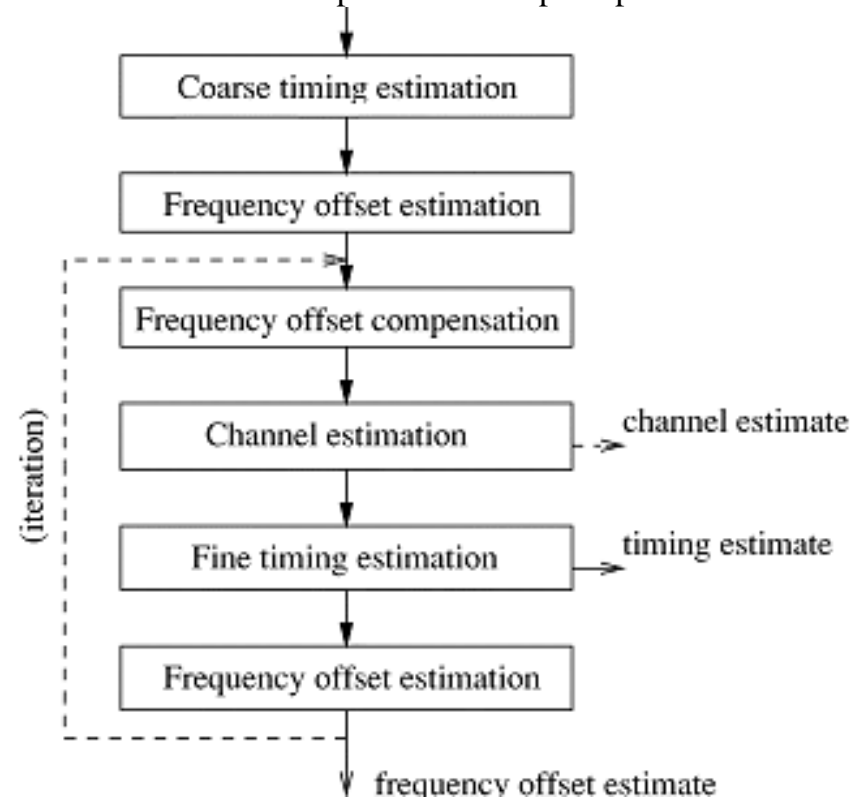

Figure 1: Synchronization using only one training symbol

\section{Chu Sequence Based Syncronization Scheme}

The synchronization is achieved using one chu sequencebased preamble which is simultaneously transmitted from all transmit antennas in the same OFDM time instant. From analysis, the chu sequence-based preamble gives better detection properties in terms of higher right timing detection probability and accurate fractional frequency synchronization even at a low SNR and in a frequency selective channel. Furthermore, application of the proposed algorithm can obtain the integral frequency offset in time domain. The synchronization is achieved by using the chu sequences which are simultaneously transmitted from the transmit antennas. At the first stage, accurate time and fractional frequency synchronization are accomplished using the good periodic correlation properties of the training symbols. At the second stage, the integral frequency offset is estimated according to its influence upon the chu sequences in time domain. The synchronization algorithm proposed shows satisfactory performance even at a low SNR.

\section{FFT-Based Frequency Offset Estimation}

In the sequel, we propose a fast and accurate FFT-based frequency offset estimation. The synchronization techniques are examined in Gaussian and Multipath fading environments for comparison. The results of the performance comparison are presented in terms of mean and mean-square error (MSE) obtained by simulations. The timing offset estimator with best performance is chosen for system timing synchronization. The FFT-based frequency offset estimation method has significantly smaller MSE than others can be performed using the same timing training symbol. Therefore, there is only one training symbol needed as preamble in whole synchronization process.

Considering a preamble consisting of two OFDM symbols, Schmidl and Cox [3] proposed a method for time and frequency synchronization respectively. Two training symbols are placed at the beginning of the frame. The first symbol has identical halves in time domain, so that the correlation between these two halves can be carried out to find out the timing metric in the receiver. However, the metric suffers from a plateau which leads some uncertainty in determining the start of the frame. To alleviate this, the authors propose a $90 \%$ averaging method to finalize the start time. At the optimum symbol time, the phase of the numerator term of the timing metric is examined, if its absolute value is less than $\pi$, the frequency offset can be estimated as a fraction of the OFDM symbol rate. Otherwise, the frequency offset will be greater than the OFDM symbol rate and the integer part of the frequency offset is estimated in a method described in section 4.2

Yun Hee Kim et al [4] made an improvement to Schmidl's method; he proposed using differentially coded training symbol to find out the integral part of the frequency offset. Therefore there is only one OFDM training symbol needed. Morelli et al [5] proposed the best linear Unbiased Estimation (BLUE) method. He also use one training symbol, but consisting of many repeated parts. It improved the frequency offset estimation range and accuracy but increased the computation. To avoid the ambiguity caused by plateau of timing metric, Minn et al [6] modified Schmidl and Cox's method and proposed two new methods. The first method uses two modifications: 1) all samples over one symbol period (excluding guard interval), are used in calculating the half symbol energy required in the timing metric and 2) the timing metrics is averaged over a window of guard interval length. The second method uses a training symbol containing four equal length parts but last two with different sign. They all give smaller estimator variance than [3], but still have large MSE in ISI channel.

Based on [6], Park et al [7] present a novel timing offset estimation method using a training symbol consisting of four parts: first two are symmetric and last two are conjugate of first two respectively, so that his

\section{Volume 6 Issue 7, July 2017 www.ijsr.net}




\section{International Journal of Science and Research (IJSR) \\ ISSN (Online): 2319-7064}

Index Copernicus Value (2015): 78.96 | Impact Factor (2015): 6.391

method produces an even sharper timing metric and has significant smaller MSE than [3] and [6]. Kanshi et al [8] proposed scheme exploits the repetitive structure of a training symbol for carrier synchronization, and presented superior performance with respect to the Schmidl approach [3] in terms of better detection properties and accuracy, and larger estimation range which is up two subcarrier spacing. Seung et al [9] proposed a timing offset estimation method and designed a new time domain preamble to give smaller MSE than other previous estimators even in the fast varying channel. Its main advantage is found in applications operating in fast Rayleigh fading channel.

In this approach, Schmidl's timing method, Minn's timing method, Park's timing method and Seung's timing method will be briefly described. And then, they will be simulated to evaluate the timing metrics. Then a simple and efficient FFT-based method for estimating the coarse and fine frequency synchronization will be proposed. Its performance will be evaluated through comparing the actual frequency offset with estimated frequency offset along with the MSE between them. This frequency estimator will also be compared with Schmidl's estimator. Finally, the best performance timing and frequency offset estimators will be integrated in a fast and efficient OFDM system synchronizer.

\section{Conclusion}

Comprehensive literature in OFDM synchronization area is presented in this review paper. Corresponding simulation results of various papers are also given for their performances evaluation. Further, a simple but efficient FFT-based frequency estimator is proposed, and compared with Schmidl's method. As shown in the simulation section, the proposed method provides good frequency estimates, and even provides better performance than method [3] in Gaussian channel and Multipath fading channel when SNR less than $12 \mathrm{~dB}$, especially in as low SNR as $0 \mathrm{~dB}$.

\section{References}

[1] H. Sari, G. Karam, and I. Jeanclaude, "Transmission techniques fordigital terrestrial TV broadcasting," IEEE Commun.Mag., vol. 33, pp.100-109, Feb. 1995.

[2] U. Reimers, "Digital video broadcasting," IEEE Commun. Mag., vol.36, pp. 104-110, June 1998.

[3] L. J. Cimini, Jr., J. C. Chuang, and N. R. Sollenberger, "Advanced cellular Internet services," IEEE Commun. Mag., vol. 36, pp. 150-159, Oct.1998.

[4] T. Pollet, M. Van Bladel, and M. Moeneclaey, "BER sensitivity ofOFDM systems to carrier frequency offset and Wiener phase noise,"IEEE Trans. Commun., vol. 43, pp. 191-193, Feb./Mar./Apr. 1995.

[5] M. Gudmundson and P. O. Anderson, "Adjacent channel interference inan OFDM system," in Proc. Vehicular Technol. Conf., Atlanta, GA, May1996, pp. 918-922.

[6] J.-J. van de Beek, M. Sandell, and P. O. Börjesson, "ML estimation oftime and frequency offset in OFDM systems," IEEE Trans. Signal Processing, vol. 45, pp. 1800-1805, July 1997.

[7] T. M. Schmidl and D. C. Cox, "Robust frequency and timing synchronization for OFDM," IEEE Trans. Commun., vol. 45, pp. 1613-1621,Dec. 1997.

[8] M. Speth, D. Daecke, and H. Meyr, "Minimum overhead burst synchronization for OFDM based broadband transmission," in Proc. GlobalTelecommun. Conf., Sydney, Australia, Nov. 1998, pp. 2777-2782.

[9] P. H. Moose, "A technique for orthogonal frequency division multiplexing frequency offset correction," IEEE Trans. Commun., vol. 42, pp.2908-2914, Oct. 1994.

[10]F. Daffara and O. Adami, "A new frequency detector for orthogonalmulticarrier transmission techniques," in Proc. Vehicular Technol. Conf.,Chicago, IL, July 1995, pp. 804-809.

[11]H. Nogami and T. Nagashima, "A frequency and timing period acquisition technique for OFDM systems," IEICE Trans. Commun., vol. E79-B,no. 8, pp. 11351146, 1996.

[12] M. Morelli and U. Mengali, “An improved frequency offset estimatorfor OFDM applications," IEEE Commun.Lett., vol. 3, pp. 75-77, Mar.1999.

[13]M. Speth, F. Classen, and H. Meyr, "Frame synchronization of OFDMsystems in frequency selective fading channels," in Proc. VehicularTechnol. Conf., Phoenix, AZ, May 1997, pp. 1807-1811.

[14] L. Hazy and M. El-Tanany, "Synchronization of OFDM systems overfrequency selective fading channels," in Proc. Vehicular Technol. Conf.,Phoenix, AZ, May 1997, pp. 2094-2098.

[15]H. Minn, M. Zeng, and V. K. Bhargava, "On timing offset estimation forOFDM systems," IEEE Commun. Lett., vol. 4, pp. 242-244, July 2000.

[16] B. Yang, K. B. Letaief, R. S. Cheng, and Z. Cao, "Timing recoveryfor OFDM transmission," IEEE J. Select. Areas Commun., vol. 18, pp.2278-2290, Nov. 2000.

[17] S. A. Fechtel and H. Meyr, "Improved frame synchronization for spontaneous packet transmission over frequency-selective radio channels,"in Proc. PIMRC, The Hague, The Netherlands, 1994, pp. 353 357.

[18]A. Czylwik, "Synchronization for single carrier modulation with frequency domain equalization," in Proc. VTC, Ottawa, ON, Canada, 1998,pp. 2277-2281.

[19]P. R. Chevillat, D. Maiwald, and G. Ungerboeck, "Rapid training ofa voiceband data-modem receiver employing an equalizer with fractional-T spaced coefficients," IEEE Trans. Commun., vol. COM-35, pp.869-876, Sept. 1987.

[20] S. H. Müller-Weinfurtner, "On the optimality of metrics for coarse framesynchronization in OFDM: A comparison," in Proc. PIMRC, Boston,MA, 1998, pp. 533-537. 